\title{
Harmonic Function Spaces of Probability Measures on Fusion Algebras
}

By

Tomohiro HAYASHI*

\begin{abstract}
In this note, we study harmonic function spaces of probability measures on fusion algebras of $C^{*}$-tensor categories and show that almost ergodicity is equivalent to ergodicity for amenable fusion algebras, which generalizes a result due to S. Popa for probability measures associated to subfactors.
\end{abstract}

\section{§. Introduction}

Nowadays fusion algebras play an important role in the index theory of subfactors. Roughly speaking, fusion algebras are a certain kind of discrete hypergroups with dimension functions. An important class of examples is provided by bimodules as follows. Let $A$ be a $\mathrm{II}_{1}$-factor and $\mathscr{C}$ be a category consisting of $A-A$ bimodules with finite index. We assume that $\mathscr{C}$ is closed under direct sum, irreducible decomposition, conjugation and unitary equivalence, and denote by $S$ the set of all unitary equivalence classes of irreducible bimodules. Then the free vector space $\mathbb{C}[S]$ over $S$ becomes a fusion algebra, where multiplication is defined by $A$-relative tensor product. The quantum dimension provides a dimension function on $S$ (see [11], [19]).

In [12], F. Hiai and M. Izumi considered random walks on fusion algebras and extended concepts of "amenability" and "ergodicity" to abstract fusion algebras. These notions were originally introduced by S. Popa for subfactors in order to classify them. He showed that amenable, ergodic (i.e., strongly amenable) subfactors have a generating property and hence they are classified by their standard invariants. The importance of these two notions can be seen also by Popa's recent announcement that standard invariant is complete for amenable subfactors ([30]).

Let $N \subset M$ be an extremal inclusion of $\mathrm{II}_{1}$-factors with finite index.

Communicated by Y. Takahashi, April 30, 1999. Revised August 9, 1999.

1991 Mathematics Subject Classification(s): 46L37

* Mathematical Institute, Tohoku University, Sendai 980-8578, Japan.

e-mail: 94m12@math.tohoku.ac.jp 
Consider the lattice of finite dimensional $C^{*}$-algebras $\left\{M_{i}^{\prime} \cap M_{j}\right\}_{i \leq j}$ where

$$
\cdots \subset M_{-2} \subset M_{-1}=N \subset M_{0}=M \subset M_{1} \subset M_{2} \subset \cdots \subset M_{\infty}
$$

is a Jones tower and a tunnel sequence. In [26] and [31] S. Popa proved the followings:

(1) The center of $N^{\prime} \cap M_{\infty}$ is either atomic or diffuse (completely non-atomic).

(2) The von Neumann algebra $\left(\bigcup_{k \geq 1} M_{-k}^{\prime} \cap M_{\infty}\right)^{\prime \prime}$ is always a factor.

(3) If $N \subset M$ has an amenable graph, almost ergodicity implies ergodicity, or equivalently, the center of $N^{\prime} \cap M_{\infty}$ is either trivial or infinite dimensional.

These facts are translated in terms of fusion algebras as follows. Let $\mathbb{C}[S]$ be the fusion algebra generated by ${ }_{N} L^{2}(M)_{N}$ and $\mu=\delta_{N} L^{2}(M)_{N}$ be the canonical probability measure on $S$ (see Section 1 for the definition of $\left.\delta_{N} L^{2}(M)_{N}\right)$. Then the above facts imply the following statements respectively:

(1) The left (or right) $\mu$-harmonic function space is either atomic or diffuse.

(2) The $(\mu, \mu)$-harmonic function space is trivial.

(3) If $\mathbb{C}[S]$ is amenable, the left (or right) $\mu$-harmonic function space is either trivial or infinite dimensional.

In this paper, we will generalize these results to arbitrary probability measures on fusion algebras of $C^{*}$-tensor categories (in particular, on fusion algebras of bimodules). The proof is simpler than Popa's original one even if we restrict our arguments to the subfactor case.

The author is grateful to Professor Shigeru Yamagami and Professor Fumio Hiai for fruitful discussions and suggestions on the present subject. He also would like to thank the referee for careful reading.

\section{§1. Basic Terminologies}

In this paper, we will freely use the notations given in $[10]\left(C^{*}\right.$-tensor categories, Frobenius duality, minimal traces, quantum dimensions, etc.). We also use the notations and the results in [12]. A tensor category consisting of $N-N$ bimodules with finite index ( $N$ being a $\mathrm{II}_{1}$-factor) has a $C^{*}$-tensor category structure in our sense (see [36] for the proof). It should be remarked that in studying fusion algebras associated with $C^{*}$-tensor categories, $C^{*}$-tensor categories may be assumed to be strict, thanks to the coherence theorem. (Any $C^{*}$-tensor category is isomorphic to a strict one. In particular, their fusion algebras are isomorphic, see [39], [40] and [10, Theorem 1.4].) Thus, throughout this paper, $C^{*}$-tensor categories are always strict, semisimple and they have conjugation and Frobenius duality. For the basic terminologies on subfactor and bimodule theory, we refer to [4], [6].

Definition (cf. [16]). Let $\mathbb{C}[S]$ be a fusion algebra with the multiplicative 
unit $I \in S$. Take two probability measures $\mu, v$ on $S$ and fix them. For a function $f \in l^{\infty}(S)$,

(1) $f$ is $(\mu, v)$-harmonic if for $s \in S$,

$$
f(s)=\sum_{t \in S} \mu * \delta_{s} * v(t) f(t)
$$

(2) $f$ is left $\mu$-harmonic if it is $\left(\mu, \delta_{I}\right)$-harmonic.

(3) $f$ is right $\mu$-harmonic if it is $\left(\delta_{I}, \mu\right)$-harmonic.

The vector space consisting of all $(\mu, v)$-harmonic functions is a closed subspace of $l^{\infty}(S)$, which is referred to as the $(\mu, v)$-harmonic function space. The same is true for the left or right harmonic function spaces.

\section{Notations.}

(1) Let $\mathscr{C}$ be a $C^{*}$-tensor category and $\mathbb{C}[S]$ be the associated fusion algebra. For each $X, Y \in \operatorname{Object}(\mathscr{C})$, we write

$$
\begin{aligned}
X Y & =X \otimes Y \\
{\left[\begin{array}{c}
X \\
Y
\end{array}\right] } & =\operatorname{Hom}(Y, X), \\
N_{X}^{Y} & =\operatorname{dim}\left[\begin{array}{l}
X \\
Y
\end{array}\right]=\operatorname{dim} \operatorname{Hom}(Y, X), \\
\delta_{X} & =d(X)^{-1} \sum_{s \in S} N_{X}^{s} d(s) \delta_{s} .
\end{aligned}
$$

(2) For a von Neumann algebra $M$, we denote its center by $Z(M)$.

\section{§2. The Relation between Harmonic Functions and Elements in $Z\left(A_{\infty}(X)\right)$}

Let $\mathscr{C}$ be a $C^{*}$-tensor category and $\mathbb{C}[S]$ be the associated fusion algebra with the unit $I \in S$. Take and fix a symmetric, generating (equivalently, nondegenerate) probability measure $\mu$ on $S$ such that $I \in \operatorname{support}(\mu)$. By using $\mu$, we can associate a von Neumann algebra $A_{\infty}(X)$ and a Hilbert space $X_{\infty}$ to an object $X$ in $\mathscr{C}$ as defined in [10] (see also [9]). For convenience, $A_{\infty}(I)$ is often denoted by $A_{\infty}$. Since the ergodicity of $\mu$ is not assumed, $A_{\infty}(X)$ may not be a factor. We summarize some properties of $A_{\infty}(X)$ and $X_{\infty}$ proved in [10]. (1) The von Neumann algebra $A_{\infty}(X)$ has a canonical tracial state $\tau_{X}\left(=\tau_{A_{\infty}(X)}\right)$, which is constructed from minimal traces and the unique tracial state on the AFD $\mathrm{II}_{1}$-factor $R$. (Sometimes we omit the symbol " $X$ ", " $A_{\infty}(X)$ " and denote a trace simply by $\tau$ if no confusion occurs.) 
(2) The Hilbert space $(X Y)_{\infty}$ is an $A_{\infty}(X)-A_{\infty}\left(Y^{*}\right)$ bimodule.

(3) $\operatorname{End}\left((X Y)_{\infty A_{\infty}\left(Y^{*}\right)}\right)=A_{\infty}(X)$.

(4) The inclusion

$$
A_{\infty}(X) \subset A_{\infty}(X Y) \subset A_{\infty}\left(X Y Y^{*}\right)
$$

is standard with the Jones projection given by $d(X)^{-1} \varepsilon_{X}^{*} \varepsilon_{X}$.

(5) There exists a one to one correspondence between the left $\mu$-harmonic function space and the center of $A_{\infty}(X)$ such that

$$
E_{A_{n}(X)}(x)=\sum_{s \in S} f(s) I_{A_{n}^{s}(X)}
$$

where $x \in Z\left(A_{\infty}(X)\right)$ (the center of $\left.A_{\infty}(X)\right)$ and $f$ is a left $\mu$-harmonic function. (" $E$ " means the trace-preserving conditional expectation.)

(6)

$$
A_{\infty} X_{\infty} \otimes_{A_{\infty}} Y_{\infty A_{\infty}} \simeq A_{\infty}(X Y)_{\infty A_{\infty}}
$$

where the left hand side is a relative tensor product with respect to the canonical trace explained in (1).

For each $n \in \mathbb{N}$, define von Neumann algebras $B_{n}$ and $B_{n}^{s}(s \in S)$ by

$$
\begin{aligned}
B_{n}= & \bigoplus_{x, y \in S^{n}}\left[\begin{array}{c}
x_{n} \ldots x_{1} X \\
y_{n} \ldots y_{1} X
\end{array}\right] \otimes_{x} R_{y} \\
& \oplus \underset{z, w \in S^{n}}{\oplus}\left[\begin{array}{c}
z_{n} \ldots z_{1} X \\
w_{n} \ldots w_{1} Y^{*}
\end{array}\right] \otimes_{z} R_{w} \\
& \oplus \underset{\alpha, \beta \in S^{n}}{\oplus}\left[\begin{array}{c}
\alpha_{n} \ldots \alpha_{1} Y^{*} \\
\beta_{n} \ldots \beta_{1} X
\end{array}\right] \otimes_{\alpha} R_{\beta} \\
& \oplus \underset{\gamma, \delta \in S^{n}}{\oplus}\left[\begin{array}{l}
\gamma_{n} \ldots \gamma_{1} Y^{*} \\
\delta_{n} \ldots \delta_{1} Y^{*}
\end{array}\right] \otimes_{\gamma} R_{\delta}
\end{aligned}
$$

and

$$
\begin{aligned}
B_{n}^{s}= & \bigoplus_{x, y \in S^{n}}\left[\begin{array}{c}
s \\
y_{n} \ldots y_{1} X
\end{array}\right] \otimes\left[\begin{array}{c}
x_{n} \ldots x_{1} X \\
s
\end{array}\right] \otimes{ }_{x} R_{y} \\
& \oplus \bigoplus_{z, w \in S^{n}}\left[\begin{array}{c}
s \\
w_{n} \ldots w_{1} Y^{*}
\end{array}\right] \otimes\left[\begin{array}{c}
z_{n} \ldots z_{1} X \\
s
\end{array}\right] \otimes{ }_{z} R_{w} \\
& \oplus \underset{\alpha, \beta \in S^{n}}{\oplus}\left[\begin{array}{c}
s \\
\beta_{n} \ldots \beta_{1} X
\end{array}\right] \otimes\left[\begin{array}{c}
\alpha_{n} \ldots \alpha_{1} Y^{*} \\
s
\end{array}\right] \otimes{ }_{\alpha} R_{\beta} \\
& \oplus \underset{\gamma, \delta \in S^{n}}{\oplus}\left[\begin{array}{c}
s \\
\delta_{n} \ldots \delta_{1} Y^{*}
\end{array}\right] \otimes\left[\begin{array}{c}
\gamma_{n} \ldots \gamma_{1} Y^{*} \\
s
\end{array}\right] \otimes{ }_{\gamma} R_{\delta}
\end{aligned}
$$


Embedding maps $B_{n} \rightarrow B_{n+1}$ are defined in the same way as the definition of $A_{n} \rightarrow A_{n+1}$ in [10, Section 3]. The minimal trace and the unique tracial state on $R$ induce a tracial state on $B_{n}$ which is compatible with the embeddings. Then the following statements can be easily checked:

(1) $B_{n} \simeq \bigoplus_{s \in S} B_{n}^{s}$ via the Frobenius reciprocity.

(2) $B_{n}^{s} \simeq R$ if $s \in \operatorname{support}\left(\mu^{n} * \delta_{X}\right) \cup \operatorname{support}\left(\mu^{n} * \delta_{Y^{*}}\right)$ and otherwise, $B_{n}^{s}$ must be $\{0\}$.

(3) For each $x \in Z\left(B_{\infty}\right)$, there exists a unique left $\mu$-harmonic function such that

$$
E_{B_{n}}(x)=\sum_{s \in S} f(s) I_{B_{n}^{s}}
$$

Conversely, for each left $\mu$-harmonic function $f$, we can find $x \in Z\left(B_{\infty}\right)$ satisfying the above equality.

(4) Let

$$
F_{n}=\sum_{x \in S^{n}, s \in S} \sum_{\rho: x_{n} \cdots x_{1} X \rightarrow s} \rho^{*} \rho \otimes e_{x} .
$$

where $\rho$ runs through the set of coisometries whose initial spaces are mutually orthogonal. Then $F_{n}=F_{m}$ for any $n, m \in \mathbb{N}$. Thus we can define $F=F_{n}$.

(5) $F B_{n} F \simeq A_{n}(X)$ and $(I-F) B_{n}(I-F) \simeq A_{n}\left(Y^{*}\right)$.

(6) Take a left $\mu$-harmonic function $f$. Let $x \in Z\left(B_{\infty}\right), y \in Z\left(A_{\infty}(X)\right)$ and $z \in Z\left(A_{\infty}\left(Y^{*}\right)\right)$ be elements corresponding to $f$. Then, under the identification of (5), we have $x F=y$ and $x(I-F)=z$.

(7) $\quad F B_{\infty} F F L^{2}\left(B_{\infty}\right)(I-F)_{(I-F) B_{\infty}(I-F)} \simeq A_{\infty}(X)(X Y)_{\infty A_{\infty}\left(Y^{*}\right)}$.

By the above consideration, we get the following proposition.

Proposition 2.1. Let $X \in \operatorname{Object}(\mathscr{C})$ and $p \in Z\left(A_{\infty}\right)$. Consider $p$ as an element of the right action of $A_{\infty}$ on $X_{\infty}$. Then we can find a unique element $p^{X} \in Z\left(A_{\infty}(X)\right)$ such that $p^{X} \xi=\xi p$ for $\xi \in X_{\infty}$. Moreover, $p$ and $p^{X}$ correspond to the same left $\mu$-harmonic function, i.e., there exists a left $\mu$-harmonic function $f$ such that

$$
E_{A_{n}}(p)=\sum_{s \in S} f(s) I_{A_{n}^{s}}, E_{A_{n}(X)}\left(p^{X}\right)=\sum_{s \in S} f(s) I_{A_{n}^{s}(X)} .
$$

Remark.

(1) In the above construction, we see that the correspondence

$$
\begin{aligned}
Z\left(A_{\infty}(X)\right) \ni x & \mapsto f(\text { a left } \mu \text {-harmonic function) } \\
& \mapsto y \in Z\left(A_{\infty}(Y)\right)
\end{aligned}
$$

is a ${ }^{*}$-isomorphism of von Neumann algebras. 
(2) Under the natural identification $L^{2}\left(A_{\infty}(X)\right) \leftrightarrow\left(X X^{*}\right)_{\infty}$, we have $J_{A_{\infty}(X)} p J_{A_{\infty}(X)}=p^{X X^{*}}$ for each $p \in Z\left(A_{\infty}\right)$, where $J_{A_{\infty}(X)}$ is a modular conjugation.

Corollary 2.2. Let $f, g$ be left $\mu$-harmonic functions and $p, q \in Z\left(A_{\infty}\right)$ be elements corresponding to $f, g$ respectively. Then for $X \in \operatorname{Object}(\mathscr{C})$, we have

$$
\tau\left(p q^{X}\right)=\lim _{n \rightarrow \infty} \sum_{s, t \in S} f(s) g(t) \mu^{n}(s) \delta_{s} * \delta_{X}(t) .
$$

Proof. Thanks to the previous proposition, we have

$$
\begin{aligned}
\tau\left(p q^{X}\right) & =\lim _{n \rightarrow \infty} \tau\left(E_{A_{n}}(p) E_{A_{n}(X)}\left(q^{X}\right)\right) \\
& =\lim _{n \rightarrow \infty} \sum_{s, t \in S} f(s) g(t) \tau\left(I_{A_{n}^{s}} \cdot I_{A_{n}^{t}(X)}\right)
\end{aligned}
$$

whereas

$$
\begin{aligned}
\tau\left(I_{A_{n}^{s}} \cdot I_{A_{n}^{t}(X)}\right) & =\sum_{x \in S^{n}} \sum_{\rho: x \rightarrow s} \sum_{\rho^{\prime}: s X \rightarrow t} \tau\left(\left(\rho^{*} \otimes 1_{X}\right) \rho^{\prime *} \rho^{\prime}\left(\rho \otimes 1_{X}\right) \otimes e_{x}\right) \\
& =\sum_{x \in S^{n}} N_{x_{n} \cdots x_{1}}^{s} N_{s X}^{t} d(t) \frac{\mu\left(x_{1}\right) \cdots \mu\left(x_{n}\right)}{d\left(x_{1}\right) \cdots d\left(x_{n}\right)} d(X)^{-1} \\
& =\mu^{n}(s) d(s)^{-1} d(X)^{-1} d(t) N_{s X}^{t} \\
& =\mu^{n}(s) \delta_{s} * \delta_{X}(t)
\end{aligned}
$$

showing the assertion.

\section{§3. Some General Properties of Harmonic Function Spaces}

In this section, we will show some properties of harmonic function spaces. The tensor category $\mathscr{C}$ is always assumed to be finitely generated.

Proposition 3.1. Let $X$ be an object in $\mathscr{C}$. If $X X^{*}$ generates the category $\mathscr{C}$, then the inclusion $A_{\infty} \subset A_{\infty}(X)$ is connected, i.e.,

$$
Z\left(A_{\infty}\right) \cap Z\left(A_{\infty}(X)\right)=\mathbb{C} .
$$

Proof. Take a projection $p \in Z\left(A_{\infty}\right) \cap Z\left(A_{\infty}(X)\right)$. Let $f$ be the left $\mu$ harmonic function corresponding to $p$. i.e.,

$$
E_{A_{n}}(p)=\sum_{s \in S} f(s) I_{A_{n}^{s}}
$$

for $n \geq 0$. Then we get

$$
p=J_{A_{\infty}(X)} p J_{A_{\infty}(X)} \in Z\left(A_{\infty}(X)\right) \cap Z\left(A_{\infty}\left(X X^{*}\right)\right) .
$$


By repeating this argument, we can prove

$$
p \in \bigcap_{n=0}^{\infty} Z\left(A_{\infty}\left(\left(X X^{*}\right)^{n}\right)\right) .
$$

Because the left $\mu$-harmonic function corresponding $J_{A_{\infty}\left(\left(X X^{*}\right)^{n}\right)} p J_{A_{\infty}\left(\left(X X^{*}\right)^{n}\right)}$ is also $f$ (here we use Proposition 2.1), we have

$$
\begin{aligned}
f(I) I & =E_{A_{0}}(p)=E_{A_{0}\left(\left(X X^{*}\right)^{2 n}\right)}(p) \\
& =\sum_{s \in S} f(s) I_{A_{0}^{s}\left(\left(X X^{*}\right)^{2 n}\right)}
\end{aligned}
$$

for any $n$. This implies that $f(I)=f(s)$ for any $s \in S$ such that $s \preceq\left(X X^{*}\right)^{2 n}$ for some $n$. Since $X X^{*}$ is a generator, $f$ must be a constant function and hence $p$ is a scalar.

Theorem 3.2. Assume that the tensor category $\mathscr{C}$ is finitely generated. Then the left $\mu$-harmonic function space is either atomic or diffuse.

Proof. Take $X \in \operatorname{Object}(\mathscr{C})$ such that $X X^{*}$ is a generator. By the remark after Proposition 2.1, we have only to show that $Z\left(A_{\infty}(X)\right)$ is either atomic or diffuse.

First we will show that the probabilistic index ([24]) of $A_{\infty}(X) \subset A_{\infty}\left(X X^{*}\right)$ is equal to $d(X)^{2}$. The proof goes by the same line of that of Pimsner-Popa inequality: Let $e=\frac{1}{d(X)} \varepsilon_{X}^{*} \varepsilon_{X} \in A_{\infty}\left(X X^{*}\right)$. Then the inclusion

$$
A_{\infty} \subset A_{\infty}(X) \subset A_{\infty}\left(X X^{*}\right)
$$

is standard with the Jones projection $e$ (but these algebras are not factors in general). Take a positive element $x \in A_{\infty}\left(X X^{*}\right)$. Thanks to a property of basic extension ([14]), the *-algebra

$$
\left\{\sum_{i} a_{i} e b_{i}: a_{i}, b_{i} \in A_{\infty}(X)\right\}
$$

is weakly dense in $A_{\infty}\left(X X^{*}\right)$. Thus, for the proof of the inequality $E_{A_{\infty}(X)}(x)$ $\geq \frac{1}{d(X)^{2}} x$, we may assume that there exist $a_{1}, \ldots, a_{n}, b_{1}, \ldots, b_{n} \in A_{\infty}(X)$ such that

$$
x=\left(\sum_{i} a_{i} e b_{i}\right)^{*}\left(\sum_{i} a_{i} e b_{i}\right)=\sum_{i, j} b_{j}^{*} E_{A_{\infty}}\left(a_{j}^{*} a_{i}\right) e b_{i} .
$$

Let $A=\left\{E_{A_{\infty}}\left(a_{i}^{*} a_{j}\right)\right\}_{i, j}$ and $P=\operatorname{diag}(e, \ldots, e)$ (a diagonal matrix) be $n \times n$ matrices. We also define a $n \times 1$ matrix $B$ by $B={ }^{t}\left(b_{1}, \ldots, b_{n}\right)$. By the 
complete positivity of $E_{A_{\infty}}$, the matrix $A$ is positive and hence there exists a matrix $C \in \operatorname{Mat}_{n}\left(A_{\infty}\right)$ such that $C^{*} C=A$. Then we compute

$$
\begin{aligned}
x & =B^{*} C^{*} C P B=B^{*} C^{*} P C B \\
& \leq B^{*} C^{*} C B=\sum_{i, j} b_{j}^{*} E_{A_{\infty}}\left(a_{j}^{*} a_{i}\right) b_{i} \\
& =d(X)^{2} E_{A_{\infty}(X)}(x) .
\end{aligned}
$$

Therefore we get the desired inequality. Since $E_{A_{\infty}(X)}(e)=\frac{1}{d(X)^{2}}$, the probabilistic index of $A_{\infty}(X) \subset A_{\infty}\left(X X^{*}\right)$ is equal to $d(X)^{2}$.

Now we will show the assertion. Let $p \in Z\left(A_{\infty}(X)\right)$ and $q \in Z\left(A_{\infty}\left(X X^{*}\right)\right)$ be the projections onto the atomic parts. If $p(I-q) \neq 0$, there exists a minimal projection $p^{\prime} \in Z\left(A_{\infty}(X) p\right)$ and a sequence of mutually orthogonal projections $\left\{q_{i}\right\}_{i=1}^{\infty}$ in $Z\left(A_{\infty}\left(X X^{*}\right)\right)$ such that $p^{\prime} q_{i} \neq 0$. This contradicts the fact that the probabilistic index of $A_{\infty}(X) \subset A_{\infty}\left(X X^{*}\right)$ is finite (see [2], [27]). Indeed, since $A_{\infty}(X) p^{\prime}$ is a factor, there exists a sequence of positive scalars $\left\{\lambda_{i}\right\}_{i=1}^{\infty}$ such that $E_{A_{\infty}(X)}\left(q_{i}\right) p^{\prime}=\lambda_{i} p^{\prime}$. Then the inequality $\sum_{i} \lambda_{l} \leq 1$ holds. On the other hand, we have $\lambda_{i} p^{\prime}=E_{A_{\infty}(X)}\left(q_{i}\right) p^{\prime} \geq d(X)^{-2} q_{i} p^{\prime}$. This implies that $\lambda_{i} \geq d(X)^{-2}$ and contradicts the inequality $\sum_{i} \lambda_{i} \leq 1$. Hence we get $p(I-q)=0$. In the same way, we also have $q\left(I-J_{A_{\infty}\left(X X^{*}\right)} p J_{A_{\infty}\left(X X^{*}\right)}\right)=0$ and $q(I-p)=0$. (Here we remark that $J_{A_{\infty}\left(X X^{*}\right)} p J_{A_{\infty}\left(X X^{*}\right)}$ is the projection onto the atomic part of $Z\left(A_{\infty}\left(X X^{*} X\right)\right)$, and the probabilistic index of $A_{\infty}\left(X X^{*}\right) \subset$ $A_{\infty}\left(X X^{*} X\right)$ is shown to be finite in the same way.) By the above consideration, we get $p=q$. Moreover, by Proposition 3.1, $Z\left(A_{\infty}(X)\right) \cap Z\left(A_{\infty}\left(X X^{*}\right)\right)=\mathbb{C}$. Therefore, $p$ is equal to 0 or $I$.

Remark. Let $N \subset M$ be a $\mathbb{I I}_{1}$-subfactor with finite index and denote its Jones tower by

$$
N \subset M \subset \cdots \subset M_{\infty}
$$

In [26] Popa proved that $Z\left(N^{\prime} \cap M_{\infty}\right) \cap Z\left(M^{\prime} \cap M_{\infty}\right)=\mathbb{C}$. (This corresponds to Proposition 3.1.) By using this fact, he also proved that $Z\left(N^{\prime} \cap M_{\infty}\right)$ is either atomic or diffuse. This implies that in the fusion algebra generated by ${ }_{N} L^{2}(M)_{N}$, the $\delta_{N} L^{2}(M)_{N}$-harmonic function space is either atomic or diffuse. Therefore, Theorem 3.2 is a generalization of his result.

Let $v$ be a probability measure on $S$ which satisfies the same assumption as $\mu$. Take a family of mutually orthogonal projections $\left\{e_{s}^{\prime}\right\}_{s \in S}$ from the AFD $\mathrm{II}_{1}$-factor $R$ such that

$$
\tau\left(e_{s}^{\prime}\right)=\frac{v(s)}{d(s)},
$$


where $\tau$ is the unique tracial state on $R$. (This is " $e_{s}$ " for $v$.) We also define a projection $e_{x}^{\prime}\left(x \in S^{n}\right)$ by replacing $\mu$ with $v$ in the definition of $e_{x}$ and set

for $x, y \in S^{n}$.

$$
{ }_{x} R_{y}^{\prime}=e_{x}^{\prime} R^{\otimes n} e_{y}^{\prime}
$$

For each $i, j \in \mathbb{N} \cup\{0\}((i, j) \neq(0,0))$, define von Neumann algebras $A_{i, j}(\mu, v)$ and $A_{i, j}^{s}(\mu, v)(s \in S)$ by

$$
\begin{gathered}
A_{i, j}(\mu, v)=\bigoplus_{x, y, z, w^{\prime} \in S^{n}}\left[\begin{array}{c}
x_{i} \cdots x_{1} z_{1} \cdots z_{j} \\
y_{i} \cdots y_{1} w_{1} \cdots w_{j}
\end{array}\right] \otimes_{x} R_{y} \otimes{ }_{z} R_{w}^{\prime}, \\
A_{i, j}^{s}(\mu, v)=\bigoplus_{x, y, z, w^{\prime} \in S^{n}}\left[\begin{array}{c}
s \\
y_{i} \cdots y_{1} w_{1} \cdots w_{j}
\end{array}\right] \otimes\left[\begin{array}{c}
x_{i} \cdots x_{1} z_{1} \cdots z_{j} \\
s
\end{array}\right] \otimes_{x} R_{y} \otimes_{z} R_{w^{\prime}}^{\prime} .
\end{gathered}
$$

For the initial algebra, we set $A_{0,0}(\mu, v)=A_{0,0}^{I}(\mu, v)=\mathbb{C}$ (hence $A_{0,0}^{s}(\mu, v)=$ $\{0\}$ if $s \neq I)$. Two embedding maps $A_{i, j}(\mu, v) \rightarrow A_{i+1, j}(\mu, v)$ and $A_{i, j}(\mu, v) \rightarrow$ $A_{i, j+1}(\mu, v)$ are defined as the definition of $A_{n} \rightarrow A_{n+1}$. The tracial state $\tau$ on $A_{i, j}(\mu, v)$ is also defined by using minimal traces and the unique tracial state on $R$ in the same way as in the construction of the tracial state on $A_{\infty}$. Then it is easy to check the followings:

(1) $A_{i, j}(\mu, v) \simeq \bigoplus_{s \in S} A_{i, j}^{s}(\mu, v)$ via the Frobenius reciprocity.

(2) $A_{i, j}^{s}(\mu, v) \simeq R$ if $s \in \operatorname{support}\left(\mu^{i} * v^{j}\right)$ and otherwise, $A_{i, j}^{s}(\mu, v)$ must be $\{0\}$.

(3) The family $\left\{A_{i, j}(\mu, v)\right\}_{i, j}$ forms commuting squares.

(4) For each $x \in Z\left(A_{\infty, j}(\mu, v)\right)$, there exists a unique left $\mu$-harmonic function $f$ such that

$$
E_{A_{l, j}(\mu, v)}(x)=\sum_{s \in S} f(s) I_{A_{t, j}^{s}(\mu, v)}
$$

Conversely, for each left $\mu$-harmonic function $f$, we can find $x \in$ $Z\left(A_{\infty, j}(\mu, v)\right)$ satisfying the above equality.

(5) For each $y \in Z\left(A_{i, \infty}(\mu, v)\right)$, there exists a unique right $v$-harmonic function $g$ such that

$$
E_{A_{l, j}(\mu, v)}(y)=\sum_{s \in S} g(s) I_{A_{t, j}^{\prime}(\mu, v)}
$$

Conversely, for each right $v$-harmonic function $g$, we can find $y \in$ $Z\left(A_{i, \infty}(\mu, v)\right)$ satisfying the above equality.

(6) For each $z \in Z\left(A_{\infty, \infty}(\mu, v)\right)$, there exists a unique $(\mu, v)$-harmonic function $h$ such that

$$
E_{A_{l, l}(\mu, v)}(z)=\sum_{s \in S} h(s) I_{A_{t, 1}^{s}(\mu, v)} .
$$

Conversely, for each $(\mu, v)$-harmonic function $h$, we can find $z \in$ $Z\left(A_{\infty, \infty}(\mu, v)\right)$ satisfying the above equality. 
Proposition 3.3. For each $f \in l^{\infty}(S), f$ is $(\mu, v)$-harmonic if and only if $f$ is both left $\mu$ - and right $\nu$-harmonic.

Proof. If $f$ is $(\mu, v)$-harmonic, then by (6) there exists $x \in Z\left(A_{\infty, \infty}(\mu, v)\right)$ such that the above equality holds. Since $E_{A_{\infty, j}(\mu, v)}(x) \in Z\left(A_{\infty, j}(\mu, v)\right)$, we can apply (4) and show that $f$ is left $\mu$-harmonic. By the same reason, $f$ is also right $v$-harmonic. The reverse implication is obvious.

Proposition 3.4. The $(\mu, \mu)$-harmonic function space consists of only constant functions.

Proof. We have only to prove that $Z\left(A_{\infty, \infty}(\mu, \mu)\right)=\mathbb{C}$, thanks to (6). Take $x \in Z\left(A_{\infty, \infty}(\mu, \mu)\right)$. Then there exists a unique $(\mu, \mu)$-harmonic function $f$ such that

$$
E_{A_{l, J}(\mu, \mu)}(x)=\sum_{s \in S} f(s) I_{A_{t, J}^{s}(\mu, \mu)}
$$

By this equality, for each $i, j$, we have

$$
\left|E_{A_{l, j}(\mu, \mu)}(x)-E_{A_{t, j+1}(\mu, \mu)}(x)\right|=\sum_{s, t \in S}|f(s)-f(t)| I_{A_{t, j}^{s}(\mu, \mu)} \cdot I_{A_{t, j+1}^{t}(\mu, \mu)} .
$$

Therefore,

$$
\begin{aligned}
\left\|E_{A_{\infty, j}(\mu, \mu)}(x)-E_{A_{\infty, j+1}(\mu, \mu)}(x)\right\|_{1} & =\lim _{i \rightarrow \infty} \sum_{s, t \in S}|f(s)-f(t)| \mu^{i+j}(s) \delta_{s} * \mu(t) \\
& =\lim _{i \rightarrow \infty} \sum_{s, t \in S}|f(s)-f(t)| \mu^{i}(s) \delta_{s} * \mu(t) .
\end{aligned}
$$

This implies that $\left\|E_{A_{\infty, j}(\mu, \mu)}(x)-E_{A_{\infty, j+1}(\mu, \mu)}(x)\right\|_{1}$ does not depend on the choice of $j$. Since the value in consideration tends to 0 as $j \rightarrow \infty$, we see that $x \in$ $A_{\infty, 0}(\mu, \mu)$. In the same way, we can also show that $x \in A_{0, \infty}(\mu, \mu)$. Combining these, we get $x \in A_{0,0}(\mu, \mu)=\mathbb{C}$, i.e., $Z\left(A_{\infty, \infty}(\mu, \mu)\right)=\mathbb{C}$.

\section{Remark.}

(1) If $\mathbb{C}[S]$ is a group algebra, statements in Proposition 3.3 and Proposition 3.4 are already known (see [16]).

(2) If $\mu=\delta_{X X^{*}}$ for some $X \in \operatorname{Object}(\mathscr{C})$, Proposition 3.4 is reduced to [31, Theorem 4.10, a)].

Corollary 3.5. If $\mathbb{C}[S]$ is commutative, $\mu$ is always ergodic.

Proof. Since $\mathbb{C}[S]$ is commutative, the $(\mu, \mu)$-harmonicity is equivalent to the left $\mu^{2}$-harmonicity. Hence by applying Proposition 3.4 , we see that $\mu^{2}$ is ergodic. Then $\mu$ is also ergodic. 
Remark. In [12] F. Hiai and M. Izumi introduced the concept of weak amenability for fusion algebras by requiring the existence of an invariant mean, and this is equivalent to the existence of a generating, symmetric and ergodic probability measure (see [10] [9]). Hence the above corollary implies that $\mathbb{C}[S]$ is weakly amenable if it is commutative. Although we consider only fusion algebras which come from tensor categories, this fact remains valid in general (see [5]).

The next corollary is useful in Section 4.

Corollary 3.6. For any left $\mu$-harmonic functions $f$ and $g$, we have

$$
\lim _{k \rightarrow \infty} \lim _{n \rightarrow \infty} \sum_{s, t \in S} f(s) g(t) \mu^{n}(s) \delta_{s} * \mu^{k}(t)=f(I) g(I)
$$

Proof. Define $x \in Z\left(A_{\infty, 0}(\mu, \mu)\right)$ and $y_{j} \in Z\left(A_{\infty, j}(\mu, \mu)\right)(j=0,1,2, \ldots)$ by

$$
\begin{aligned}
& E_{A_{t, 0}(\mu, \mu)}(x)=\sum_{s \in S} f(s) I_{A_{i, 0}^{s}(\mu, \mu)}, \\
& E_{A_{l, j}(\mu, \mu)}\left(y_{j}\right)=\sum_{s \in S} g(s) I_{A_{i, j}^{s}(\mu, \mu)} .
\end{aligned}
$$

Take an ultrafilter $\omega$. Because $\left\{y_{j}\right\}_{j}$ is a norm-bounded sequence, it converges weakly to some element in $Z\left(A_{\infty, \infty}(\mu, \mu)\right)=\mathbb{C}$ as $j \rightarrow \omega$, whereas

$$
\tau\left(y_{j}\right)=\tau\left(E_{A_{0, j}(\mu, \mu)}\left(y_{j}\right)\right)=\sum_{s \in S} g(s) \mu^{j}(s)=g(I) .
$$

Thus $\left\{y_{j}\right\}_{j}$ converges weakly to $g(I)$ as $j \rightarrow \omega$. Since $\omega$ is arbitrary, we also have $y_{j} \rightarrow g(I)$ weakly as $j \rightarrow \infty$. This implies

$$
\begin{aligned}
\lim _{k \rightarrow \infty} \lim _{n \rightarrow \infty} \sum_{s, t \in S} f(s) g(t) \mu^{n}(s) \delta_{s} * \mu^{k}(t) & =\lim _{k \rightarrow \infty} \tau\left(x y_{k}\right) \\
& =\tau(x) g(I)=f(I) g(I) .
\end{aligned}
$$

\section{§4. The Equivalence between Almost Ergodicity and Ergodicity in Amenable Fusion Algebras}

Let $N \subset M$ be an extremal $\mathrm{II}_{1}$-subfactor of finite index and amenable graph with the Jones tower denoted by $N \subset M \subset M_{1} \subset \cdots \subset M_{\infty}$. In [31, Corollary 6.4], Popa proved that "the center $Z\left(N^{\prime} \cap M_{\infty}\right)$ is either trivial or infinite dimensional" by using his characterization of amenability. Equivalently, under the assumption of amenability, if $\operatorname{dim} Z\left(N^{\prime} \cap M_{\infty}\right)<\infty$ (he called this condition almost ergodicity), then $Z\left(N^{\prime} \cap M_{\infty}\right)$ must be one dimensional (ergodicity). This fact implies that the harmonic function space of 
$\delta_{N} L^{2}(M)_{N}$ is either trivial or infinite dimensional if the fusion algebra generated by ${ }_{N} L^{2}(M)_{N}$ is amenable. The aim of this section is to extend this fact to arbitrary probability measures.

Let $\mathscr{C}$ be a $C^{*}$-tensor category and $\mathbb{C}[S]$ be the associated fusion algebra. Let $\mu$ be a symmetric, generating probability measure on $S$ such that $\operatorname{support}(\mu)$ $\ni I$. We assume that the left harmonic function space of $\mu$ is finite dimensional and denote its dimension by $n_{0}$. Then $Z\left(A_{\infty}\right)$ is finite dimensional and we denote a basis of $Z\left(A_{\infty}\right)$ consisting of minimal projections by $\left\{p_{1}, p_{2}, \ldots, p_{n_{0}}\right\}$. For each $p_{i}$, there exists a unique left $\mu$-harmonic function $f_{i}$ such that

$$
E_{A_{n}}\left(p_{i}\right)=\sum_{s \in S} f_{i}(s) I_{A_{n}^{s}}
$$

(Here we remark that $\tau\left(p_{i}^{s}\right)=\tau\left(E_{A_{0}(s)}\left(p_{i}^{s}\right)\right)=f_{i}(s)$ for any $s \in S$.) We also assume that $\mathscr{C}$ is finitely generated.

Definition 4.1. For each $X \in \operatorname{Object}(\mathscr{C})$, we define $n_{0} \times n_{0}$ matrices as follows:

$$
\begin{aligned}
& M(X)(i, j)=d\left(A_{\infty} p_{i} p_{i} X_{\infty} p_{j_{A_{\infty} p_{j}}}\right), \\
& \Delta(X)(i, j)=\left[{ }_{A_{\infty} p_{l}} p_{i} X_{\infty} p_{j_{A_{\infty} p_{J}}}\right]^{1 / 2}, \\
& L(X)(i, j)=\operatorname{dim}_{A_{\infty} p_{l}} p_{i} X_{\infty} p_{j}, \\
& R(X)(i, j)=\operatorname{dim} p_{i} X_{\infty} p_{j_{A_{\infty} p_{j}}},
\end{aligned}
$$

where $d(\cdot)$ denotes the quantum dimension (the square root of the minimal index $),[\cdot]$ means Jones index, and "dim" is the coupling constant. Here we recall that the Jones index $[X]$ of an $A-B$ bimodule $X$ is given by

$$
[X]=\left(\operatorname{dim}_{A} X\right) \cdot\left(\operatorname{dim} X_{B}\right) .
$$

Lemma 4.2. For each $X \in \operatorname{Object}(\mathscr{C})$,

(1) $L(X)(i, j)=\frac{\tau\left(p_{i}^{X^{*}} p_{j}\right)}{\tau\left(p_{i}\right)} d(X)=\frac{d(X)}{\tau\left(p_{i}\right)} \lim _{n \rightarrow \infty} \sum_{s, t \in S} f_{j}(s) f_{i}(t) \mu^{n}(s) \delta_{s} * \delta_{X^{*}}(t)$.

(2) $\Delta(X)(i, j)=\left(\frac{\tau\left(p_{i}^{X^{*}} p_{j}\right) \tau\left(p_{j}^{X} p_{i}\right)}{\tau\left(p_{i}\right) \tau\left(p_{j}\right)}\right)^{1 / 2} d(X)^{2}$.

Proof. First we introduce "the commutant of traces" according to [35]. Let $N$ be a $\mathrm{II}_{1}$-factor with the unique tracial state $\tau$ and $N$ acts on a Hilbert space $H$. By a general property of normal representations (see [Takesaki's book]), there exist an index set $J$ and a projection $p \in N$ such that

$$
{ }_{N} H \simeq{ }_{N} L^{2}(N) \oplus \# J(1 \oplus \cdots \oplus 1 \oplus p) .
$$


Then $N^{\prime}$ can be represented by the right multiplication of elements in

$$
\operatorname{diag}(1, \ldots, 1, p) \cdot \operatorname{Mat}_{\# J}(N) \cdot \operatorname{diag}(1, \ldots, 1, p)
$$

and hence the restriction of $\tau \otimes \operatorname{Tr}\left(\operatorname{Tr}\right.$ means the usual trace on $\left.\mathrm{Mat}_{\# J}(\mathbb{C})\right)$ on this algebra defines a trace on $N^{\prime}$. We call this trace the commutant of $\tau$ and denote it by $\tau^{\prime}$. Then we have

$$
\operatorname{dim}_{N f^{\prime}} f^{\prime} H=\tau^{\prime}\left(f^{\prime}\right)
$$

for any projection $f^{\prime} \in N^{\prime}$.

Now we will show the equality $(1)$. Take $Y \in \operatorname{Object}(\mathscr{C})$ and consider the left module $A_{\infty} p_{i} p_{i}\left(Y Y^{*}\right)_{\infty}$. Let $e=d(Y)^{-1} \varepsilon_{Y}^{*} \varepsilon_{Y}$ and $\tau^{\prime}=\left(\tau_{A_{\infty} p_{l}}\right)^{\prime}\left(A_{\infty} p_{i}\right.$ is a $\mathrm{II}_{1}$-factor). Since $e p_{i}\left(Y Y^{*}\right)_{\infty}=p_{i} L^{2}\left(A_{\infty}\right)$, we get $\tau^{\prime}\left(e p_{i}\right)=1$. On the other hand, we compute

$$
\tau_{A_{\infty}\left(Y Y^{*}\right)^{o p}}\left(e p_{i}\right)=\tau_{A_{\infty}\left(Y Y^{*}\right)}\left(e p_{i}^{Y Y^{*}}\right)=\tau_{A_{\infty}\left(Y Y^{*}\right)}\left(e p_{i}\right)=d(Y)^{-2} \tau\left(p_{i}\right) .
$$

Thanks to the factoriality of $A_{\infty}\left(Y Y^{*}\right)^{o p} p_{i}$, a trace on it is unique up to scalar multiples. Hence we get

$$
\tau^{\prime}=\frac{d(Y)^{2}}{\tau\left(p_{i}\right)} \tau_{A_{\infty}\left(Y Y^{*}\right)^{o p}}
$$

on $A_{\infty}\left(Y Y^{*}\right)^{o p} p_{i}$.

Let $Y=I \oplus X$. In the canonical way, $X_{\infty}$ and $X_{\infty}^{*}$ can be regarded as submodules of $\left(Y Y^{*}\right)_{\infty}$. Let $f$ be a projection from $\left(Y Y^{*}\right)_{\infty}$ onto $X_{\infty}$. By the property of left dimensions, we have

$$
\begin{aligned}
L(X)(i, j) & =\tau^{\prime}\left(p_{i} p_{j}^{Y Y^{*}} f\right)=\frac{d(Y)^{2}}{\tau\left(p_{i}\right)} \tau_{A_{\infty}\left(Y Y^{*}\right)^{o p}}\left(p_{i} p_{j}^{Y Y^{*}} f\right) \\
& =\frac{d(Y)^{2}}{\tau\left(p_{i}\right)} \tau_{A_{\infty}\left(Y Y^{*}\right)}\left(p_{i}^{Y Y^{*}} p_{j} J_{A_{\infty}(Y)} f J_{A_{\infty}(Y)}\right) .
\end{aligned}
$$

Here we remark that $J_{A_{\infty}(Y)} f J_{A_{\infty}(Y)}$ is a projection from $\left(Y Y^{*}\right)_{\infty}$ onto $X_{\infty}^{*}$ and we can consider $J_{A_{\infty}(Y)} f J_{A_{\infty}(Y)} A_{\infty}\left(Y Y^{*}\right) J_{A_{\infty}(Y)} f J_{A_{\infty}(Y)}=A_{\infty}\left(X^{*}\right)$. Under this identification, it is shown that

and

$$
p_{i}^{Y Y^{*}} p_{j} J_{A_{\infty}(Y)} f J_{A_{\infty}(Y)}=p_{i}^{X^{*}} p_{j}
$$

$$
\left.d(Y)^{2} \tau_{A_{\infty}\left(Y Y^{*}\right)}\right|_{A_{\infty}\left(X^{*}\right)}=d\left(X^{*}\right) \tau_{A_{\infty}\left(X^{*}\right)}
$$

Hence we get

$$
L(X)(i, j)=\frac{d(Y)^{2}}{\tau\left(p_{i}\right)} \cdot \frac{d(X)}{d(Y)^{2}} \tau_{A_{\infty}\left(X^{*}\right)}\left(p_{i}^{X^{*}} p_{j}\right)=\frac{\tau\left(p_{i}^{X^{*}} p_{j}\right)}{\tau\left(p_{i}\right)} d(X)
$$

showing (1). The relation (2) is a direct consequence of (1). 
Lemma 4.3. For each $X, Y \in \operatorname{Object}(\mathscr{C})$, the following statements hold:

(1) $M(X \oplus Y)=M(X)+M(Y)$.

(2) $M(X Y)=M(X) M(Y)$.

(3) $M\left(X^{*}\right)={ }^{t} M(X)$.

(4) $\Delta(X Y)(i, j) \geq(\Delta(X) \Delta(Y))(i, j)$.

(5) $\Delta(X)(i, j) \geq M(X)(i, j)$.

(6) $\Delta(X)(i, j)=(L(X)(i, j) R(X)(i, j))^{1 / 2}$.

(7) $R(X)(i, j)=L\left(X^{*}\right)(j, i)$.

Proof. Thanks to the additivity and the multiplicativity of quantum dimensions ([19], [11]), (1) and (2) are easily shown by using the isomorphism

$$
A_{\infty} p_{l} p_{i}(X Y)_{\infty} p_{j_{A_{\infty} p_{j}}} \simeq \oplus_{k A_{\infty} p_{t}} p_{i} X_{\infty} p_{k} \otimes A_{\infty} p_{k} p_{k} Y_{\infty} p_{j_{A_{\infty} p_{j}}} .
$$

The equality (3) is also trivial from the conjugation-invariance of quantum dimensions.

For the proof of (4), we use the following well-known (and obvious) fact: Let $N$ be a $\mathrm{II}_{1}$-factor and ${ }_{N} X_{N},{ }_{N} Y_{N}$ be two $N$ - $N$ bimodules with finite index. Then we have the inequality

$$
\left[{ }_{N} X_{N} \oplus{ }_{N} Y_{N}\right]^{1 / 2} \leq\left[{ }_{N} X_{N}\right]^{1 / 2}+\left[{ }_{N} Y_{N}\right]^{1 / 2} .
$$

Finally, (5), (6) and (7) are immediate from definitions.

Lemma 4.4. For each $X \in \operatorname{Object}(\mathscr{C})$ such that $X X^{*}$ is a generator, the following inequality holds:

$$
\left\|L_{X X}\right\| \leq\left\|M\left(X X^{*}\right)\right\| \leq\left\|\Delta\left(X X^{*}\right)\right\| \leq d(X)^{2}
$$

where $L_{X X^{*}}$ is the left regular representation of $X X^{*}$ on $l^{2}(S)$ (see the definition of amenability of fusion algebras in [10, Definition 2.1]), i.e.,

$$
L_{X X} \delta_{s}=\sum_{t \in S} N_{X X^{*} s}^{t} \delta_{t}
$$

Proof. First we will show that $M\left(X X^{*}\right)$ is an irreducible matrix. For each $i$, set

$$
\Omega_{i}=\left\{j: p_{i} p_{j}^{\left(X X^{*}\right)^{n}} \neq 0 \text { for some } n \in \mathbb{N}\right\} .
$$

If $\Omega_{i} \neq\left\{1, \ldots, n_{0}\right\}, p_{\Omega_{l}}=\sum_{j \in \Omega_{l}} p_{j}$ is not equal to $I$. On the other hand, it is easy to see that $p_{\Omega_{1}}$ is an element in $\bigcap_{n \in \mathbb{N}} Z\left(A_{\infty}\left(\left(X X^{*}\right)^{n}\right)=\mathbb{C}\right.$ (by Proposition 3.1). This is a contradiction. Hence we have $\Omega_{i}=\left\{1, \ldots, n_{0}\right\}$. If $p_{i} p_{j}^{\left(X X^{*}\right)^{n}}$ $\neq 0$, then $p_{i}\left(X X^{*}\right)_{\infty}^{n} p_{j} \neq 0$ and $M\left(X X^{*}\right)^{n}(i, j) \neq 0$. This implies that $M\left(X X^{*}\right)$ is irreducible. (Here we remark that by the same reason, $\Delta\left(X X^{*}\right), L\left(X X^{*}\right)$ and $R\left(X X^{*}\right)$ are also irreducible.)

By the irreducibility and self-adjointness, we can apply the PerronFrobenius theorem to get 


$$
\begin{aligned}
\left\|M\left(X X^{*}\right)\right\| & =\lim _{n \rightarrow \infty}\left\{M\left(X X^{*}\right)^{n}(1,1)\right\}^{1 / n} \\
& =\lim _{n \rightarrow \infty}\left\{\sum_{s \in S} N_{\left(X X^{*}\right)^{n}}^{s} M(s)(1,1)\right\}^{1 / n} \\
& \geq \lim _{n \rightarrow \infty}\left(N_{\left(X X^{*}\right)^{n}}^{I}\right)^{1 / n}=\left\|L_{X X^{*}}\right\|
\end{aligned}
$$

and

$$
\begin{aligned}
\left\|\Delta\left(X X^{*}\right)\right\| & =\lim _{n \rightarrow \infty}\left\{\Delta\left(X X^{*}\right)^{n}(1,1)\right\}^{1 / n} \\
& \leq \lim _{n \rightarrow \infty}\left\{\Delta\left(\left(X X^{*}\right)^{n}\right)(1,1)\right\}^{1 / n} \\
& =\limsup _{n \rightarrow \infty}\left(\frac{\tau\left(p_{1}^{\left(X X^{*}\right)^{n}} p_{1}\right)}{\tau\left(p_{1}\right)}\right)^{1 / n} d(X)^{2} \leq d(X)^{2} .
\end{aligned}
$$

In the rest of this section, we always assume that $\mathscr{C}$ is amenable.

Corollary 4.5. For each $X \in \operatorname{Object}(\mathscr{C})$, we have

$$
\Delta(X)=M(X) .
$$

Proof. Let $Y$ be an object such that $Y Y^{*}$ is a generator. Then by the previous lemma, we have

$$
\left\|L_{Y Y^{*}}\right\| \leq\left\|M\left(Y Y^{*}\right)\right\| \leq\left\|\Delta\left(Y Y^{*}\right)\right\| \leq d(Y)^{2} .
$$

Moreover, thanks to the amenability, $\left\|L_{Y Y^{*}}\right\|=d(Y)^{2}$ holds. Hence we get $\left\|M\left(Y Y^{*}\right)\right\|=\left\|\Delta\left(Y Y^{*}\right)\right\|$. By the Perron-Frobenius theorem, this equality and Lemma 4.3 (5) imply that $M\left(Y Y^{*}\right)=\Delta\left(Y Y^{*}\right)$. This relation is equivalent to the extremality of each bimodule $A_{\infty} p_{t} p_{i}\left(Y Y^{*}\right)_{\infty} p_{j_{A_{\infty} p_{j}}}$, i.e., the subfactor

$$
A_{\infty} p_{i} \subset \operatorname{End}\left(p_{i}\left(Y Y^{*}\right)_{\infty} p_{j_{A_{\infty} p_{j}}}\right)
$$

is extremal. By this fact, we can show that the bimodule ${ }_{A_{\infty} p_{l}} p_{i} X_{\infty} p_{j_{A_{\infty}} p_{l}}$ is also extremal for any object $X$. Indeed, for each $X$, there exists a generator $Y Y^{*}$ such that $X \preceq Y Y^{*}$. Then

$$
A_{\infty} p_{t} p_{i} X_{\infty} p_{j_{A_{\infty}} p_{J}} \preceq A_{\infty} p_{t} p_{i}\left(Y Y^{*}\right)_{\infty} p_{j_{A_{\infty} p_{j}}},
$$

showing that $A_{\infty} p_{t} p_{i} X_{\infty} p_{j_{A_{\infty}} p_{J}}$ is also extremal.

The extremality of ${ }_{A_{\infty} p_{l}} p_{i} X_{\infty} p_{j_{A_{\infty}} p_{J}}$ is equivalent to

$$
M(X)(i, j)=\Delta(X)(i, j),
$$

whence we get $M(X)(i, j)=\Delta(X)(i, j)$ for any $X$. 
Lemma 4.6. The number

$$
\alpha_{i, j}=\frac{R(X)(i, j)}{L(X)(i, j)}
$$

does not depend on the choice of $X$ whenever $L(X)(i, j) \neq 0$ and satisfies

$$
\alpha_{i, j} \cdot \alpha_{j, k}=\alpha_{i, k}, \quad \alpha_{i, j}^{-1}=\alpha_{j, i}
$$

Proof. Thanks to the extremality of ${ }_{A_{\infty} p_{l}} p_{i} X_{\infty} p_{j_{A_{\infty} p_{j}}}$, for each subbimodule

$$
A_{\infty} p_{l} H_{A_{\infty} p_{j}} \preceq A_{\infty} p_{l} p_{i} X_{\infty} p_{j_{A_{\infty}} p_{j}},
$$

we have

$$
\frac{\operatorname{dim} H_{A_{\infty} p_{J}}}{\operatorname{dim}_{A_{\infty} p_{l}} H}=\frac{\operatorname{dim} p_{i} X_{\infty} p_{j_{A_{\infty} p_{J}}}}{\operatorname{dim}_{A_{\infty} p_{l}} p_{i} X_{\infty} p_{j}} .
$$

By this property and the fact that $X_{\infty}$ and $Y_{\infty}$ are submodule of $(X \oplus Y)_{\infty}$ for any $X, Y \in \operatorname{Object}(\mathscr{C})$, we get the first assertion. The second assertion follows from

$$
A_{\infty} p_{l} p_{i} X_{\infty} p_{j_{A_{\infty}} p_{J}} \otimes_{A_{\infty} p_{J} A_{\infty} p_{j}} p_{j} Y_{\infty} p_{k A_{\infty} p_{k}} \preceq A_{\infty} p_{l} p_{i}(X Y)_{\infty} p_{k A_{\infty} p_{k}}
$$

and the extremality of these bimodules. The last assertion is a consequence of the property

$$
\operatorname{dim}_{A_{\infty} p_{t}} H=\operatorname{dim} H_{A_{\infty} p_{l}}^{*} .
$$

\section{Lemma 4.7.}

(1) The family of matrices $\{\Delta(X)\}_{X \in \mathrm{Object}(\mathscr{C})}$ has a common Perron-Frobenius eigenvector

$$
\gamma=\left(\gamma_{1}, \ldots, \gamma_{n_{0}}\right)
$$

$\left(\gamma_{i}>0\right)$ such that

$$
\Delta(X) \gamma=d(X) \gamma
$$

(2) For each $X \in \operatorname{Object}(\mathscr{C})$,

$$
\|\Delta(X)\|=d(X) .
$$

Proof. Take a generator $X_{0}=X X^{*}$ and fix it. Let $\gamma=\left(\gamma_{1}, \ldots, \gamma_{n_{0}}\right)$ $\left(\gamma_{i}>0,\|\gamma\|_{2}=1\right)$ be the Perron-Frobenius eigenvector of $\Delta\left(X_{0}\right)$. For any irreducible object $s$, there exists $n \in \mathbb{N}$ such that $s \preceq X_{0}^{n}$. Let $Y=X_{0}^{n} \ominus s$. $\left(s \oplus Y=X_{0}^{n}.\right) \quad$ Then we get

$$
\begin{aligned}
(\Delta(s)+\Delta(Y)) \gamma & =\Delta\left(X_{0}^{n}\right) \gamma=d\left(X_{0}\right)^{n} \gamma \\
& =d(s) \gamma+d(Y) \gamma,
\end{aligned}
$$


while we have

$$
\begin{aligned}
d(s)+d(Y) & =\|d(s) \gamma+d(Y) \gamma\|_{2}=\|(\Delta(s)+\Delta(Y)) \gamma\|_{2} \\
& \leq\|\Delta(s)\|+\|\Delta(Y)\| \leq d(s)+d(Y)
\end{aligned}
$$

where the last inequality follows from $\|\Delta(Z)\|^{2}=\left\|\Delta\left(Z Z^{*}\right)\right\|$ and $\left\|\Delta\left(Z Z^{*}\right)\right\| \leq$ $d(Z)^{2}$ (see Lemma $4.2(2)$ ). Hence we get

$$
\begin{gathered}
\|\Delta(s) \gamma\|_{2}=\|\Delta(s)\|=d(s), \\
\|\Delta(Y) \gamma\|_{2}=\|\Delta(Y)\|=d(Y), \\
\|\Delta(s) \gamma+\Delta(Y) \gamma\|_{2}=\|\Delta(s) \gamma\|_{2}+\|\Delta(Y) \gamma\|_{2} .
\end{gathered}
$$

The last equality implies that there exists a scalar $a>0$ such that $a \Delta(s) \gamma=$ $\Delta(Y) \gamma$. Then we have

$$
(1+a) \Delta(s) \gamma=(\Delta(s)+\Delta(Y)) \gamma=(d(s)+d(Y)) \gamma
$$

and

$$
a \cdot d(s)=\|a \Delta(s) \gamma\|_{2}=\|\Delta(Y) \gamma\|_{2}=d(Y) .
$$

These imply that $\Delta(s) \gamma=d(s) \gamma$ and we get (1). The equality (2) is a consequence of $(1)$ and $\|\Delta(X)\| \leq d(X)$.

We are now ready to prove the following theorem.

Theorem 4.8. Assume that $\mathscr{C}$ is amenable. Let $\mu$ be a symmetric generating probability measure on $S$ such that $I \in \operatorname{support}(\mu)$. Then the left $\mu$-harmonic function space is either trivial or infinite dimensional.

Proof. We have only to prove that, if the left $\mu$-harmonic function space is finite dimensional, it is one-dimensional. We continue to use the notations as above. Recall that $p_{i} \in Z\left(A_{\infty}\right)$ corresponds to a left $\mu$-harmonic function $f_{i}$. For each probability measure $v$ on $S$, define an $n_{0} \times n_{0}$ matrix $L_{v}$ by

$$
L_{v}(i, j)=\sum_{s \in S} \frac{v(s)}{d(s)} L(s)(i, j)
$$

We remark that $L(s)(i, j)$ actually depends on $\mu$. By Lemma 4.2, we get

$$
\begin{aligned}
L_{v}(i, j) & =\frac{1}{\tau\left(p_{i}\right)} \lim _{n \rightarrow \infty} \sum_{s, t, u \in S} v(s) f_{j}(t) f_{i}(u) \mu^{n}(t) \delta_{t} * \delta_{s}(u) \\
& =\frac{1}{\tau\left(p_{i}\right)} \lim _{n \rightarrow \infty} \sum_{t, u \in S} f_{j}(t) f_{i}(u) \mu^{n}(t) \delta_{t} * v(u) .
\end{aligned}
$$

This expression, together with the relation $L_{v^{k}}=\left(L_{v}\right)^{k}$, enables us to show that 


$$
\begin{aligned}
L_{\mu}^{k}(i, j) & =L_{\mu^{k}}(i, j) \\
& =\frac{1}{\tau\left(p_{i}\right)} \lim _{n \rightarrow \infty} \sum_{t, u \in S} f_{j}(t) f_{i}(u) \mu^{n}(t) \delta_{t} * \mu^{k}(u) \\
& \rightarrow \tau\left(p_{j}\right)
\end{aligned}
$$

as $k \rightarrow \infty$. (Here we use Corollary 3.6.) For each probability measure $v$ on $S$, define

$$
\Delta_{v}(i, j)=\sum_{s \in S} \frac{v(s)}{d(s)} \Delta(s)(i, j)
$$

Then we compute

$$
\begin{aligned}
\Delta_{\mu}^{k}(i, j) & =\sum_{s \in S} \frac{\mu^{k}(s)}{d(s)} \Delta(s)(i, j) \\
& =\sum_{s \in S} \frac{\mu^{k}(s)}{d(s)} \alpha_{i, j}^{1 / 2} L(s)(i, j)=\alpha_{i, j}^{1 / 2} L_{\mu}^{k}(i, j) .
\end{aligned}
$$

Thus $\Delta_{\mu}^{k}(i, j)$ tends to $\alpha_{i, j}^{1 / 2} \tau\left(p_{j}\right)$ as $k \rightarrow \infty$. On the other hand, by the definition of $\alpha_{i, j}$, we have

$$
\alpha_{i, j}=\lim _{k \rightarrow \infty} \frac{L_{\mu}^{k}(j, i)}{L_{\mu}^{k}(i, j)}=\frac{\tau\left(p_{i}\right)}{\tau\left(p_{j}\right)}
$$

and hence

$$
\Delta_{\mu}^{k}(i, j) \rightarrow\left(\tau\left(p_{i}\right) \tau\left(p_{j}\right)\right)^{1 / 2} .
$$

This implies that the common Perron-Frobenius eigenvector $\gamma$ obtained in Lemma 4.7 is proportional to $\left(\tau\left(p_{1}\right)^{1 / 2}, \ldots, \tau\left(p_{n_{0}}\right)^{1 / 2}\right)$. Therefore, for each $s \in S$,

$$
\begin{aligned}
d(s) \tau\left(p_{i}\right)^{1 / 2} & =\sum_{j} \Delta(s)(i, j) \tau\left(p_{j}\right)^{1 / 2} \\
& =\sum_{j} \alpha_{i, j}^{1 / 2} L(s)(i, j) \tau\left(p_{j}\right)^{1 / 2} \\
& =\sum_{j}\left(\frac{\tau\left(p_{i}\right)}{\tau\left(p_{j}\right)}\right)^{1 / 2} \frac{\tau\left(p_{i}^{s^{*}} p_{j}\right)}{\tau\left(p_{i}\right)} d(s) \tau\left(p_{j}\right)^{1 / 2} \\
& =d(s) \frac{\tau\left(p_{i}^{s^{*}}\right)}{\tau\left(p_{i}\right)^{1 / 2}},
\end{aligned}
$$


and we have $\tau\left(p_{i}\right)=\tau\left(p_{i}^{s^{*}}\right)$. This is equivalent to the relation $f_{i}(I)=f_{i}\left(s^{*}\right)$. Since $s$ is arbitrary, each $f_{i}$ must be a constant function, showing that $\mu$ is ergodic.

As a direct application, we get the following result due to S. Popa ([31, Corollary 6.4]).

Corollary 4.9. Let $N \subset M$ be a $I I_{1}$-subfactor with finite index and amenable graph. If we denote the associated Jones tower by

$$
N \subset M \subset M_{1} \subset \cdots \subset M_{\infty},
$$

then the center of $N^{\prime} \cap M_{\infty}$ is either trivial or infinite dimensional.

Proof. Let $X={ }_{N} L^{2}(M)_{N}$ and $\mathscr{C}$ be a $C^{*}$-tensor category generated by $X$. (Thus objects of $\mathscr{C}$ are $N-N$ bimodules.) The associated fusion algebra is described by $\mathbb{C}[S]$. The amenability of the principal graph means that $\mathscr{C}$ is amenable ([12, Proposition 2.4 (4)]). Hence we can apply the above theorem to show that the harmonic function space of $\delta_{X}$ is either trivial or infinite dimensional.

\section{Remark.}

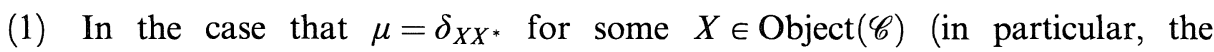
subfactor case), we can work with Ocneanu's path-space construction of bimodules to get the result in Theorem 4.8: We just repeat our arguments by replacing as follows:

$$
\begin{aligned}
A_{n}(Y) \leftrightarrow\left[\begin{array}{c}
\left(X X^{*}\right)^{n} Y \\
\left(X X^{*}\right)^{n} Y
\end{array}\right] \\
Z_{n} \leftrightarrow\left[\begin{array}{c}
\left(X X^{*}\right)^{n} Z \\
\left(X X^{*}\right)^{n}
\end{array}\right] .
\end{aligned}
$$

(2) If we remove the assumption of amenability, Theorem 4.8 does not hold. In fact, there exists a counter example constructed by U. Haagerup. See [12, Example 8.11].

In the group case, Theorem 4.8 holds without the assumption of amenability. More strongly, in [15] it is shown that the Poisson boundary is either trivial or diffuse. Here we can present a direct proof of this fact by using our methods as follows.

Proposition 4.10. Let $G$ be a countable discrete group ( $G$ may not be amenable) with the (algebraic) group algebra $\mathbb{C}[G]$. Let $\mu$ be a symmetric, generating probability measure on $G$ such that $I \in \operatorname{support}(\mu)$, where $I \in G$ is a unit. Then the left $\mu$-harmonic function space is either trivial or diffuse. 
Proof. Let $\alpha: G \rightarrow \operatorname{Aut}(R)$ ( $R$ is an AFD $\mathrm{II}_{1}$-factor) be an outer action. Then $\left\{{ }_{R} L^{2}(R) \alpha_{g_{R}}\right\}_{g \in G}$ generates a $C^{*}$-tensor category and the associated fusion algebra is $\mathbb{C}[G]$. Assume that the left $\mu$-harmonic function space is atomic. It suffices to show that this space is trivial. We use the notations in this section. Remark that the left $\mu$-harmonic function space may be infinite-dimensional in this case. Since $d(g)=1$ for any $g \in G$, we get $A_{\infty}(g)=A_{\infty}$. From this, for each $g \in G$, there exists $i \in\left\{1, \ldots, n_{0}\right\}$ such that $p_{1}^{g}=p_{i}$. Hence we have $f_{1}(g) \in\left\{f_{i}(I)\right\}_{i}$ for any $g \in G$. Consider the set of all values of $f_{1}$ and denote it by $\Omega$. Then the inclusion $\Omega \subset\left\{f_{i}(I)\right\}_{i}$ holds. On the other hand, since $\Omega$ is countable, we can write $\Omega=\left\{a_{n}\right\}_{n=1}^{\infty}$. Consequently,

$$
\sum_{n} a_{n} \leq \sum_{i} f_{i}(I)=\sum_{i} \tau\left(p_{i}\right)=1
$$

By this inequality and the positivity of $f_{1}, f_{1}$ attains its maximum. Hence there exists $g_{0} \in G$ such that $f_{1}\left(g_{0}\right)=\left\|f_{1}\right\|_{\infty}$. Then for each integer $n$, we have

$$
\begin{aligned}
f_{1}\left(g_{0}\right) & =\sum_{g \in G} \mu^{n} * \delta_{g_{0}}(g) f_{1}(g) \\
& \leq \sum_{g \in G} \mu^{n} * \delta_{g_{0}}(g) f_{1}\left(g_{0}\right)=f_{1}\left(g_{0}\right) .
\end{aligned}
$$

This implies that $f_{1}(g)=f_{1}\left(g_{0}\right)$ if $\mu^{n} * \delta_{g_{0}}(g) \neq 0$. Since $n$ is arbitrary and $\mu$ is symmetric, generating and $\mu(I)>0, f_{1}$ must be a constant function. By the same argument, all $f_{i}$ must be constant, proving the triviality of the left $\mu$ harmonic function space.

Final remark. Although we consider fusion algebras coming from $C^{*}$ tensor categories (in particular, bimodules), there exist some fusion algebras which do not admit a $C^{*}$-tensor category structure (in particular, they cannot be realized by bimodules). Fusion algebras of this kind are dealt with in [34], for example. Our methods established in this paper heavily depend on the properties of a $C^{*}$-tensor category and we do not know whether the present results remain true or not for fusion algebras which do not come from $C^{*}$-tensor categories.

\section{References}

[1] Bisch, D., Bimodules, higher relative commutants and the fusion algebra associated to a subfactor, in "Operator Algebras and Their applications", eds. P. A. Fillmore and J. A. Mingo, Field Inst. Commun., 13 (1997), 16-63.

[2] Ballet, M., Denizeau, Y. and Havet, J.-F., Indice d'une esperance conditionelle, Compositio Math., 66 (1988), 199-236. 
[3] Bisch, D. and Haagerup, U., Composition of subfactors: new examples of infinite depth subfactors, Ann. Sci. Ec. Norm. Sup., 29 (1996), 329-383.

[4] Evans, D. and Kawahigashi, Y., Quatum Symmetry on Operator Algebras, Oxford Univ. Press, 1998.

[ 5 ] Gebuhrer, M. O., Remarks on amenability of discrete hypergroups, in "Harmonic Analysis and Discrete Potential Theory", ed. M. A. Picardello, Plenum Press, New York, 1992, pp. 131-143.

[6] Goodman, F. M. P. de la Harpe and Jones, V., Coxeter Graphs and Towers of Algebras, Springer-Verlag, 1989.

[ 7 ] Haagerup, U., Talk at conferences “Operator Algebras and Applications”, Geneva, 1994.

[ 8 ] Hayashi, T., Factoriality of harmonic traces on string algebras, to appear in Internat. Jour. Math.

[9] - Realization of amenable bicategories and some properties of probability measures on fusion algebras, to appear in Jour. Math. Soc. of Japan.

[10] Hayashi, T. and Yamagami, S., Amenable tensor category and their realizations as AFD bimodules, to appear in Jour. Funct. Anal.

[11] Hiai, F., Minimizing indices of conditional expectations onto a subfactor, Publ. RIMS, Kyoto Univ., 24 (1988), 673-678.

[12] Hiai, F. and Izumi, M., Amenability and strong amenability for fusion algebras with applications to subfactor theory, Internat. Jour. Math., 9 (1998), 669-722.

[13] Izumi, M., Application of fusion rules to classification of subfactors, Publ. RIMS, Kyoto Univ., 27 (1991), 953-994.

[14] Jones, V., Index for subfactors, Invent. Math., 72 (1983), 1-25.

[15] Kaimanovich, V., The Poisson boundary of covering Markov operators, Israel J. Math., 89 (1995), 77-134.

[16] — Bi-harmonic functions on groups, C. R. Acad. Sci. Paris. Serie I, 314 (1992), 259264.

[17] Kosaki, H., Extension of Jones' index theory on index to arbitrary subfactors, J. Funct. Anal., 109 (1986), 123-140.

[18] Longo, R., Index of subfactors and statistics of quantum fields, II, Comm. Math. Phys., 130 (1990), 285-239.

[19] - Minimal index and braided subfactors, J. Funct. Anal., 109 (1992), 98-112.

[20] Longo, R. and Roberts, J., A theory of dimension, K-Theory, 11 (1997), 103-159.

[21] MacLane, S., Categories for Working Mathematicians 2nd edition, Springer-Verlag, BerlinNew York, 1998.

[22] Ocneanu, A., Quantized groups, string algebras and Galois theory for algebras, in "Operator Algebras and Applications", Vol 2, eds. D. E. Evans and M. Takesaki, London Math. Soc. Lect. Note Ser., 136 (1988), 119-172.

[23] —, Quantum Symmetry, Differential Geometry of Finite Graphs and Classification of Subfactors, Univ. of Tokyo Seminary Notes 45, Notes recorded by Y. Kawahigashi, 1991.

[24] Pimsner, M. and Popa, S., Entropy and index for subfactors, Ann. Sci. Eco. Norm. Sup., 19 (1986), 57-106.

[25] Popa, S., Classification of subfactors: the reduction to commuting squares, Invent. Math., 101 (1990), 19-43.

[26] — Classification of amenable subfactor of type II, Acta Math., 172 (1994), 163-255.

[27] — Classification of subfactors and their endomorphisms, CBMS Lecture Notes, 86 (1995).

[28] - Symmetric enveloping algebras, amenability and AFD properties for subfactors, Math. Res. Lett., 1 (1994), 409-425. 
[29] Popa, S., An axiomatization of the lattice of higher relative commutants of a subfactor, Invent. Math., 120 (1995), 427-445.

[30] - Amenability in the theory of subfactors, in "Operator Algebras and Quantum Field Theory", International Press, eds. S. Doplicher et al, 199-211.

[31] - Some properties of the symmetric enveloping algebra of a subfactor, with applications to amenability and property $\mathrm{T}$, preprint.

[32] Sauvageot, J. L., Sur le produit tensoriel relatif d'espaces de hilbert, J. Oper. Theory, 9 (1983), 237-252.

[33] Sunder, V. S., $\mathrm{II}_{1}$ factors, their bimodules and hypergroups, Trans. Amer. Math. Soc., 330 (1992), 227-256.

[34] Tambara, D. and Yamagami, S., Tensor categories with fusion rules of self-duality for finite abelian groups, Jour. of Alg., 209 (1998), 692-707.

[35] Yamagami, S., A report on Ocneanu's lecture. Investigations concerning group actions on operator algebras and index entropy, (Japanese) (Kyoto, 1991), RIMS Kokyuroku, 839 (1993), 20-62.

[36] —, A note on Ocneanu's approach to Jones' index theory, Internat. Jour. Math., 4 (1993), 859-871.

[37] —, Frobenius reciprocity in tensor categories, to appear in Math. Scand.

[38] - Notes on amenability of commutative fusion algebras, preprint.

[39] - Frobenius duality in $C^{*}$-tensor categories, in preparation.

[40] —, Tensor category for operator algebraists, manuscripts. 